(1)

CrossMark

\title{
Social stigma in the time of coronavirus disease 2019
}

\author{
Giovanni Sotgiu (10 ${ }^{1}$ and Claudia C. Dobler (1) ${ }^{2,3}$
}

Affiliations: ${ }^{1}$ Dept of Medical, Surgical and Experimental Sciences, University of Sassari, Sassari, Italy. ${ }^{2}$ Institute for Evidence-Based Healthcare, Bond University, Robina, Queensland, Australia. ${ }^{3}$ Dept of Respiratory and Sleep Medicine, Liverpool Hospital, Sydney, New South Wales, Australia.

Correspondence: Giovanni Sotgiu, Clinical Epidemiology and Medical Statistics Unit, Dept of Medical, Surgical and Experimental Sciences, University of Sassari, Sassari, Italy. E-mail: gsotgiuduniss.it

Addressing COVID-19-related stigma https://bit.ly/2CPuxO1

Cite this article as: Sotgiu G, Dobler CC. Social stigma in the time of coronavirus disease 2019. Eur Respir J 2020; 56: 2002461 [https://doi.org/10.1183/13993003.02461-2020].

The coronavirus disease 2019 (COVID-19) pandemic has dramatically changed the lives of people around the globe since it appeared in Wuhan, China, at the beginning of December 2019. The burden of disease and its death toll have had an unprecedented impact on the healthcare, economic and financial systems of low-, middle- and high-income countries [1-3]. Peoples' lives have been disrupted and negatively impacted by COVID-19-related suffering and lockdowns at community and household level.

The rigidity of lockdown measures has radically changed social interactions, with virtual meetings replacing face-to-face meetings to reduce the risk of severe acute respiratory syndrome coronavirus 2 (SARS-CoV-2) transmission. Social distancing (maintaining a physical distance of, for example, $\geqslant 1 \mathrm{~m}$ ), besides frequent hand washing, use of face masks in public and increasing ventilation in indoor spaces, is one of the most important health behaviours to reduce virus transmission from one infectious patient to others $[4,5]$. While there is mounting evidence that SARS-CoV-2 is transmitted by the inhalation of airborne particles [6], there are a number of unanswered questions regarding virus transmission, including the risk of transmission from asymptomatic individuals and contact with contaminated inanimate surfaces on which SARS-CoV-2 can survive for prolonged periods $[7,8]$.

Insufficient knowledge and contradictory information about the transmission of SARS-CoV-2 and protective measures, such as wearing face masks in public, is associated with anxiety among the population. People's uncertainty and anxiety has led them to believe biased and vague information provided by traditional media, social media (e.g. Twitter, Facebook, Instagram, etc.) and self-proclaimed experts [9]. Misinformation about COVID-19 has rapidly spread worldwide (occasionally exceeding the speed of spread of the actual COVID-19 pandemic).

During the early days of the pandemic, the identification of infectious clusters, super spreaders (those who are responsible for infecting a large number of people) or community outbreaks caused widespread fear among the public. It was speculated that COVID-19 was as contagious as measles and was associated with a very high case fatality rate. Images broadcast on television showing military trucks transporting coffins of COVID-19 victims were reminiscent of the deadly Western Africa Ebola virus epidemic a few years earlier.

Anxiety caused by lockdowns, many unknowns around COVID-19 and the fear of being infected have given rise to stigma in local communities. A "witch hunt" hysteria developed worldwide fuelling 
discrimination and attacks against vulnerable people. COVID-19 index cases and other infected persons together with their close contacts identified through the public health activity of contact tracing, have been blamed and compared to criminals. There have been media reports from around the globe describing how frontline healthcare workers have been assaulted, spit on, hit with rocks, sprayed with bleach, denied rides to work and made homeless because of fears that they would transmit SARS-CoV-2 to the people around them. There is anecdotal evidence and some media reports that Chinese people have fallen victim to racist attacks during the COVID-19 pandemic and that Chinese restaurants around the world are at risk of shutting down completely due to a decrease in customers and because of discrimination against Chinese restaurants and lockdowns.

Social stigma was defined by Erving Goffman in 1963 as "an attribute which is deeply discrediting" that reduces a person "from a whole and usual person to a tainted, discounted one" $[10,11]$. It creates a dichotomy between "being normal and acceptable" versus "being tainted and undesirable". Social stigma is commonly related to race, culture, sex, intelligence and health. The conceptualisation of stigma identifies four elements that interact with each other: anticipated, perceived, experienced, and internalised stigma [12]. COVID-19 has been associated with all of these elements of social stigma. People have modified their actions because of fear of being discriminated against, for example by avoiding testing for SARS-CoV-2 (anticipated stigma); patients and their families felt judged by others (perceived stigma); infected or exposed persons were excluded, isolated and discriminated against by their household and/or community members (experienced stigma); and some patients might have felt shame and self-rejection (internalised stigma). Persons infected with SARS-CoV-2 may experience intersecting (multiple) stigmas, for example when they also belong to a marginalised ethnic group. Social stigma negatively affects social justice, as stigmatised people feel that they cannot actively participate in society. The three core elements of social justice are agency (the capacity of individuals to act independently and to make their own free choices), respect and association (the capacity to connect and participate) [13].

Social stigma, discrimination and exclusion have been described in detail in other infectious diseases (e.g. tuberculosis, HIV/AIDS) [14-17]. Stigmatising language (e.g. "tuberculosis suspect") that has been criticised by advocates, has also been used during the current pandemic (e.g. "COVID-19 suspect"). Such judgmental terms have the power to influence attitudes and behaviours, for example by preventing patients from seeking treatment or by influencing the way in which policy-makers view and seek to address a disease.

The anxiety and concern of being discriminated against can lead to two hazardous clinical and public health consequences: delayed presentation of symptomatic patients to healthcare services (prognostic deterioration) and under-detection of infectious individuals (increased viral transmission to susceptible contacts). Delayed diagnosis has been associated with more severe disease, mainly in the elderly and vulnerable groups, while delayed notification of an infectious patient can facilitate the rapid spread of SARS-CoV-2 in the community [18].

People with greater personal resources (income, education, social support) and good mental health have been shown to have more knowledge about emerging infectious diseases, be less worried and be less likely to stigmatise $[19,20]$. Education, clear and honest communication and the use of non-discriminatory language have the potential to significantly improve the knowledge, attitudes and behaviours related to COVID-19 and reduce social stigma [21]. Effective communication includes expert information about the disease (e.g. contagiousness, number of diagnosed people, fatality rate, seroprevalence in the community, indicating the proportion of people who have been infected at some point in the past, etc.) and recommended infection control measures. National, regional and local healthcare services that communicate transparently and work reliably and efficiently can also alleviate fears among the community and reduce stigmatisation and social discrimination. An example of a successful initiative to counteract misconceptions, misinformation and stigma is the "Trinità health educational model" [22]. The initiative was implemented in a small Sardinian town, where, after a local COVID-19 outbreak, the mayor and the main political party decided to use an interactive educational programme based on the World Health Organization (WHO) principles of health education [23]. The local population had an opportunity to interact with an expert online and to get answers to their questions, which helped to address general and specific concerns about COVID-19.

The infodemic - characterised by an overabundance of news, mixing facts, rumours and fake news - is a key driver of social stigma in our time [24]. Conspiracy theories that COVID-19 is spread via 5G cell towers or that Bill Gates caused the epidemic to sell the world a vaccine, and false (preventive) treatments promoted on social media such as eating garlic or drinking bleach can hinder the fight against COVID-19 and can even have fatal consequences. The WHO uses their information network EPI-WIN to address the infodemic by identifying real-time evidence and misinformation and creates actionable and behavioural 
change messages in response (for example, the myth buster series). Google removes misleading information about COVID-19 from its platforms and Twitter fact-checks tweets and adds a warning message to provide additional context and information if a tweet is flagged.

New and effective educational approaches are needed to counteract the damaging effects of the infodemic during COVID-19 and to increase empathy towards population groups at risk of stigmatisation [25].

Conflict of interest: G. Sotgiu has nothing to disclose. C.C. Dobler has nothing to disclose.

\section{References}

1 Donthu N, Gustafsson A. Effects of COVID-19 on business and research. J Bus Res 2020; 117: 284-289.

2 Proaño CR. On the macroeconomic and social impact of the coronavirus pandemic in Latin America and the developing world. Inter Econ 2020; 55: 159-162.

3 Nava S, Tonelli R, Clini EM. An Italian sacrifice to the COVID-19 epidemic. Eur Respir J 2020; 55: 2001445.

4 Chu DK, Akl EA, Duda S, et al. Physical distancing, face masks, and eye protection to prevent person-to-person transmission of SARS-CoV-2 and COVID-19: a systematic review and meta-analysis. Lancet 2020; 395: 1973-1987.

5 MacIntyre CR, Wang Q. Physical distancing, face masks, and eye protection for prevention of COVID-19. Lancet 2020; 395: 1950-1951.

6 Zhang $\mathrm{R}$, Li Y, Zhang AL, et al. Identifying airborne transmission as the dominant route for the spread of COVID-19. Proc Natl Acad Sci 2020; 117: 14857-14863.

7 Liu Z, Chu R, Gong L, et al. The assessment of transmission efficiency and latent infection period on asymptomatic carriers of SARS-CoV-2 infection. Int J Infect Dis 2020; in press [https://doi.org/10.1016/j.ijid.2020. 06.036].

8 Widders A, Broom A, Broom J. SARS-CoV-2: the viral shedding vs infectivity dilemma. Infect Dis Health 2020; in press [https://doi.org/10.1016/j.idh.2020.05.002].

9 Malecki K, Keating JA, Safdar N. Crisis communication and public perception of COVID-19 risk in the era of social media. Clin Infect Dis 2020; in press [https://doi.org/10.1093/cid/ciaa758].

10 Goffman E. Stigma: notes on the management of spoiled identity. Englewood Cliffs, Prentice Hall, 1963.

11 Dowdy DW, Zwerling AA, Stennett A, et al. Measuring stigma to assess the social justice implications of health-related policy decisions: application to novel treatment regimens for multidrug-resistant tuberculosis. $M D M$ Policy Pract 2020; 5: 2381468320915239.

12 Kane JC, Elafros MA, Murray SM, et al. A scoping review of health-related stigma outcomes for high-burden diseases in low-and middle-income countries. BMC Med 2019; $17: 17$.

13 Bailey TC, Merritt MW, Tediosi F. Investing in justice: ethics, evidence, and the eradication investment cases for lymphatic filariasis and onchocerciasis. Am J Public Health 2015; 105: 629-636.

14 Daftary A, Frick M, Venkatesan N, et al. Fighting TB stigma: we need to apply lessons learnt from HIV activism. BMJ Glob Health 2017; 2: e000515.

15 Daftary A. HIV and tuberculosis: the construction and management of double stigma. Soc Sci Med 2012; 74: 1512-1519.

16 Datiko DG, Jerene D, Suarez P. Stigma matters in ending tuberculosis: nationwide survey of stigma in Ethiopia. BMC Public Health 2020; 20: 190.

17 Zachariah R, Harries AD, Srinath S, et al. Language in tuberculosis services: can we change to patient-centred terminology and stop the paradigm of blaming the patients? Int J Tuberc Lung Dis 2012; 16: 714-717.

18 Rong XM, Yang L, Chu HD, et al. Effect of delay in diagnosis on transmission of COVID-19. Math Biosci Eng 2020; 17: 2725-2740.

19 Des Jarlais DC, Galea S, Tracy M, et al. Stigmatization of newly emerging infectious diseases: AIDS and SARS. Am J Public Health 2006; 96: 561-567.

20 Des Jarlais DC, Stuber J, Tracy M, et al. Social factors associated with AIDS and SARS. Emerging Infect Dis 2005; 11: 1767-1769.

21 Gollust SE, Nagler RH, Fowler EF. The emergence of COVID-19 in the U.S.: a public health and political communication crisis. J Health Polit Policy Law 2020; in press [https://doi.org/10.1215/03616878-8641506].

22 Sotgiu G, Carta G, Suelzu L, et al. How to demystify COVID-19 and reduce social stigma. Int J Tuberc Lung Dis 2020; 24: 640-642.

23 World Health Organization. Health education: theoretical concepts, effective strategies and core competencies: a foundation document to guide capacity development of health educators. https://apps.who.int/iris/handle/10665/ 119953

24 Naeem SB, Bhatti R. The Covid-19 'infodemic': a new front for information professionals. Health Info Libr J 2020; in press [https://doi.org/10.1111/hir.12311].

25 UNICEF, World Health Organization, International Federation of Red Cross, Red Crescent Societies. Social Stigma associated with COVID-19. https://www.unicef.org/documents/social-stigma-associated-coronavirus-disease-covid-19 